\title{
Oxygen derived free radicals and synovial fluid hyaluronate
}

\author{
Herkko Saari
}

\begin{abstract}
High performance liquid chromatography with TSK 5000 PW or TSK 6000 PW size exclusion columns combined with a ${ }^{125}$ I labelled hyaluronic acid binding protein assay was used to study the effects of oxygen derived free radicals on synovial fluid hyaluronate. A continuous flux of free radicals was generated by the xanthine oxidase/hypoxanthine system. When the free radical flux was generated with xanthine oxidase/hypoxanthine in the presence of the iron chelator desferrioxamine and the hydroxyl radical scavenger mannitol a $30-50 \%$ decrease in hyaluronate peak was detected, but the molecular weight of synovial fluid hyaluronate remained almost unchanged as a result of reaction with superoxide radicals and hydrogen peroxide. When trace amounts of iron and EDTA were present in the reaction mixture depolymerisation of synovial fluid hyaluronate occurred, and it reached a final molecular weight of about 13500 daltons. These results suggest that superoxide and hydroxyl radicals may have a different mode of action on synovial fluid hyaluronate. Superoxide radicals and hydrogen peroxide do not induce depolymerisation but, rather, change the molecular configuration of synovial fluid hyaluronate.
\end{abstract}

Oxygen derived free radicals are produced by polymorphonuclear leucocytes through a membrane bound enzyme, NADPH oxidase, ${ }^{12}$ and can be produced in vitro by xanthine oxidase or by autoxidation of ferrous iron. ${ }^{34}$ Xanthine oxidase reduces molecular oxygen to superoxide anion by oxidising hypoxanthine to urate. In the presence of trace amounts of iron superoxide radicals and hydrogen peroxide undergo the Haber-Weiss reaction producing extremely reactive hydroxyl radicals, which react rapidly with almost every type of molecule close to their site of formation. ${ }^{5}$ This may cause depolymerisation of hyaluronate and a decrease in synovial fluid viscosity. ${ }^{6}$ It has been suggested that in inflammatory joint diseases oxygen derived free radicals are responsible for the depolymerisation of hyaluronate and the resulting decrease in viscosity of the synovial. ${ }^{7}$ The effect of oxygen derived free radicals on hyaluronate has been previously interpreted from studies on changes in the viscosity of either purified hyaluronate or synovial fluid. ${ }^{8}$ Although the viscosity is greatly altered by the radical reactions, it is a complex function of hyaluronate concentration, ionic strength, and the presence of other molecules capable of interacting with hyaluronate. Greenwald et al have shown a decrease in the hydrodynamic volume of human umbilical cord hyaluronate by triggered polymorphonuclear neutrophilic leucocytes. ${ }^{8}$

In this paper the effect of various oxygen derived free radicals on synovial fluid hyaluronate was studied by high performance liquid chromatography (HPLC) with size exclusion column and a ${ }^{125}$ I labelled hyaluronic acid binding protein ( $\left.{ }^{125} \mathrm{I}-\mathrm{HABP}\right)$ assay to determine simultaneously the synovial fluid hyaluronate concentration and the degree of polymerisation.

\section{Patients and methods}

SAMPLES

Non-inflammatory synovial fluid samples were obtained from patients with meniscus lesion. After aspiration into a syringe the samples were immediately centrifuged at $1000 \mathrm{~g}$ for 10 minutes to obtain pelleted cells. Synovial fluid supernatants were stored at $-20^{\circ} \mathrm{C}$ without preservatives.

HIGH PERFORMANCE LIQUID CHROMATOGRAPHY High performance liquid chromatography was performed with an LKB 2150 HPLC pump equipped with Toyo Soda TSK 5000 PW (30 $\mathrm{cm} \times 7.5 \mathrm{~mm})$ or TSK $6000 \mathrm{PW}(60 \mathrm{~cm} \times 7.5$ $\mathrm{mm})$ size exclusion columns. An LKB 2150 variable monitor with $\mathrm{LKB} 2220$ recording integrator was used for detection of synovial fluid hyaluronate at a wavelength of $206 \mathrm{~nm}$. The elution buffer was $50 \mathrm{mM}$ sodium phosphate, $\mathrm{pH} 6.5$, at a continuous flow of $0.5-1.0$ $\mathrm{ml} / \mathrm{min}$. The interassay variation coefficient for the retention time of high molecular weight hyaluronate was $2 \cdot 3 \%$. In general, the retention times of hyaluronate peaks were within 20 seconds if care was taken to dilute the samples appropriately before injection (usually 1/100 with the elution buffer).

GENERATION OF OXYGEN DERIVED FREE RADICALS Oxygen derived free radicals were generated in the reaction mixture containing $0.5 \mathrm{ml}$ synovial fluid, $0.2 \mathrm{mM}$ hypoxanthine, and $0.16 \mathrm{U} / \mathrm{ml}$ xanthine oxidase. The reaction was started by adding xanthine oxidase, and the suspension was kept aerobic by constant mechanical stirring. The appropriate control experiments with corresponding HPLC were performed in the absence of a radical generation system. All incubations were at room temperature. To obtain maximal hydroxyl radical production in
Unioninkatu 38 , SF-00170 Helsinki, Finland

Accepted for publication 20 March 1990 
some of the experiments indicated $50 \mu \mathrm{M}$ ferrichloride and $50 \mu \mathrm{M}$ EDTA were also present to catalyse the iron dependent HaberWeiss reaction. In experiments requiring prevention of hydroxyl radical formation $1 \mathrm{mM}$ desferrioxamine and $80 \mathrm{mM}$ mannitol were added to the reaction mixture. For HPLC a sample of the reaction mixture was diluted $1 / 100$ with $50 \mathrm{mM}$ sodium phosphate buffer and $50 \mu \mathrm{l}$ was injected into the chromatographic apparatus.

\section{RADIOMETRIC DETECTION OF SYNOVIAL FLUID} HYALURONATE

For radiometric detection of hyaluronate from fractions collected during chromatography, the ${ }^{125}$ I-HABP assay was used (Pharmacia Diagnostica AB, Uppsala, Sweden). Radioactivity of the samples was counted with an LKB 1975 RiaGamma automatic gammacounter.

CALIBRATION OF THE HPLC COLUMNS Molecular weight standards (Healon) were obtained from Pharmacia Diagnostica AB. Molecular weight determination of the standards was performed by the manufacturer using a low angle laser light scattering method. Each standard was chromatographed separately. To obtain adequate molecular weight resolution the standards were diluted to a hyaluronate concentration of $10 \mu \mathrm{g} / \mathrm{ml}$. A calibration curve was obtained for the TSK $5000 \mathrm{PW}$ column with a flow of $0.5 \mathrm{ml} / \mathrm{min}$. Calibration of the TSK 6000 PW column was performed as described previously. ${ }^{9}$

\section{MEASUREMENT OF VISCOSITIES}

Relative viscosities of the synovial fluids were measured at $37^{\circ} \mathrm{C}$ in $2 \mathrm{ml}$ samples by rotation viscometry with four different shear rates. After stabilisation for one minute the viscosity value was read.

\section{CHEMICALS}

Superoxide dismutase (EC 1.15.1.1, $4300 \mathrm{U} / \mathrm{ml}$ ), catalase (EC 1.11.1.16, $2800 \mathrm{U} / \mathrm{ml}$ ) from bovine liver, and xanthine oxidase (EC 1.2.3.2) from buttermilk were obtained from Sigma Chemical Company, St Louis, MO. Hypoxanthine was from Merck, Darmstadt, FRG.

\section{Results}

The depolymerisation of synovial fluid hyaluronate caused by hydroxyl radicals was seen on repeated HPLC as a time dependent gradual shift of the hyaluronate peak in the direction of smaller molecular size (fig 1A). Finally, the depolymerisation product was masked behind the large peak containing most of the protein fraction of synovial fluid. During degradation the height of the hyaluronate peak was unaltered, but the molecular size decreased. Samples were taken from the reaction mixture every $\mathbf{3 0}$ minutes under constant oxygen derived free radical flux.
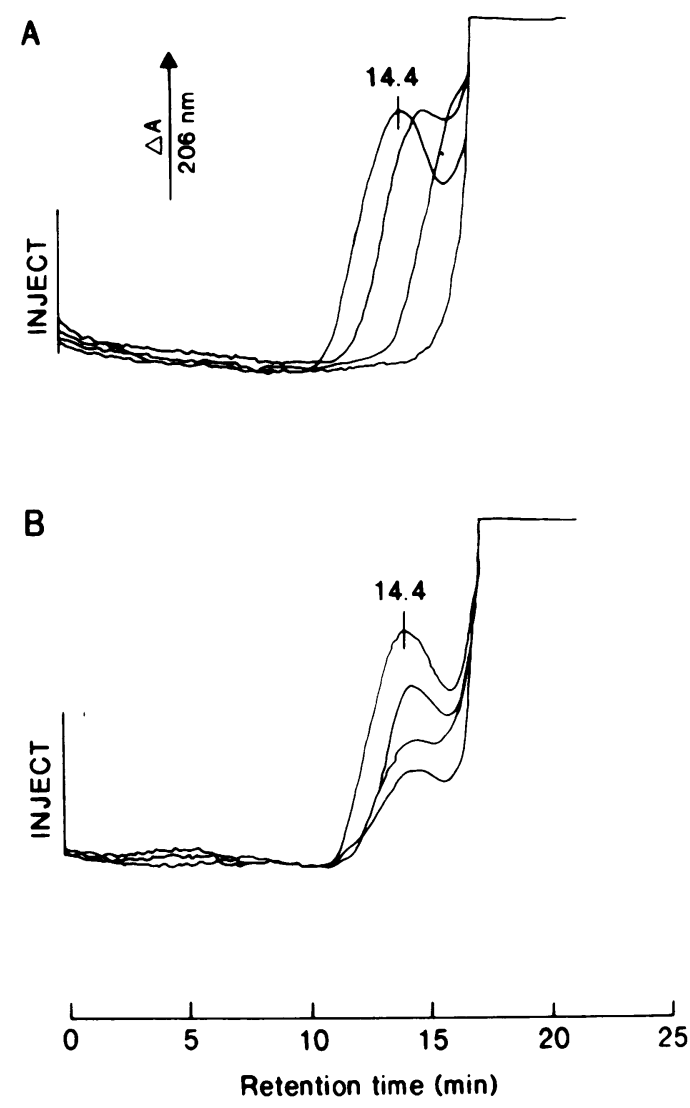

Figure 1 Time dependence of the depolymerisation of symovial fluid hyaluronate under constant oxygen derived free radicals flux produced by xanthine oxidase/hypoxanthine in the presence of $(A)$ irom and EDTA and $(B)$ desferrioxamine and mannitol.

In contrast, superoxide radicals and hydrogen peroxide caused a considerable decrease in hyaluronate without changes in the retention time (Fig 1B). To produce superoxide and prevent the formation of hydroxyl radicals 1 $\mathrm{mM}$ desferrioxamine and $40 \mathrm{mM}$ mannitol were used to abolish the trace amount of iron present in synovial fluid samples and in the reagents used and to scavenge the hydroxyl radicals respectively. Under these conditions only superoxide radicals and hydrogen peroxide arising through spontaneous dismutation are formed.

The molecular size of the free radical depolymerisation product of synovial fluid hyaluronate was studied further by the following experiment: $200 \mu \mathrm{l}$ fractions of HPLC elution buffer were collected during the HPLC run at the time intervals indicated in figs $2 \mathrm{~A}, \mathrm{~B}$, and $\mathrm{C}$, and their hyaluronate content was determined by a specific radiometric test using ${ }^{125}$ I labelled hyaluronate binding protein. In normal, highly viscous synovial fluid the hyaluronate peak in both HPLC and the ${ }^{125}$ I-HABP assay occurred at the same retention time of 14.2 minutes, indicating high molecular weight hyaluronate.

If, instead, the ${ }^{125} \mathrm{I}$-HABP assay was performed on fractions collected during HPLC after degradation of the synovial fluid hyaluronate with hydroxyl radicals produced by the xanthine oxidase/hypoxanthine system in the presence of $50 \mu \mathrm{M} \mathrm{FeCl}$ and $50 \mu \mathrm{M}$ EDTA the hyaluronate peak eluted at a retention time of 

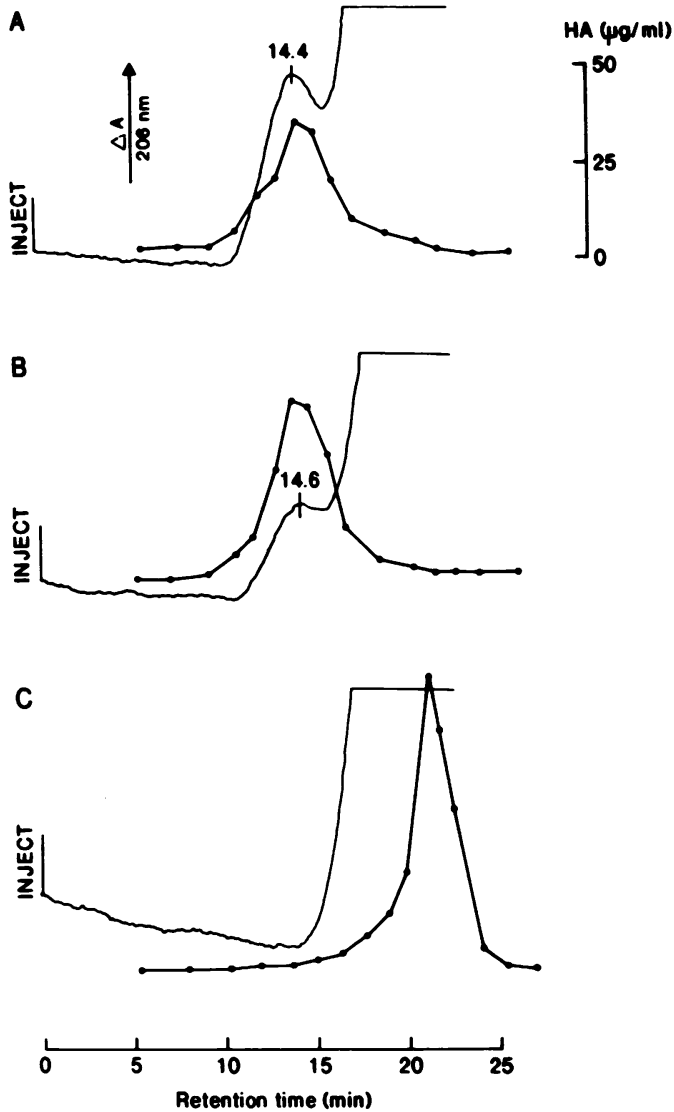

Figure 2 High performance liquid chromatograms and simultaneous detection of hyaluronate $(H A)$ with ${ }^{125} I$ labelled hyaluronate binding protein of fractions collected during chromatography. (A) Native synovial fluid from a meniscus patient; $(B)$ effect of superoxide radicals produced in the xanthine oxidaselhypoxanthine system in the presence of $1 \mathrm{mM}$ desferrioxamine and $80 \mathrm{mM}$ mannitol; (C) effect of hydroxyl radicals produced in the xanthine

oxidase/ hypoxanthine system in the presence of $50 \mu \mathrm{M} \mathrm{FeCl}$ and $50 \mu M$ EDTA. Chromatography was performed with $a$ TSK $6000 \mathrm{PW}$ column and a flow of $1.0 \mathrm{ml} / \mathrm{min}$.

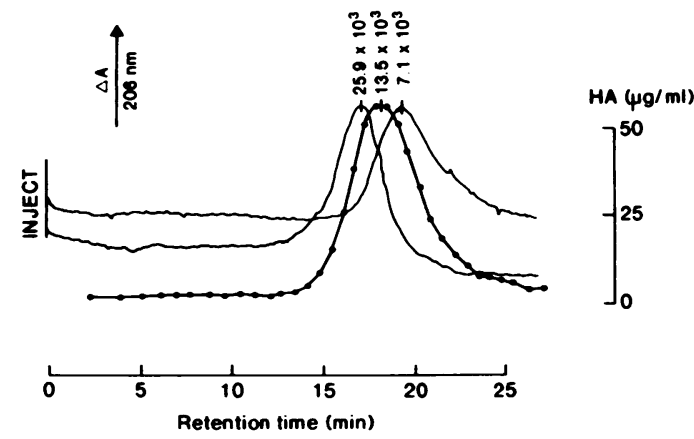

Figure 3 Molecular weight of the final deploymerisation product after exposure of synovial fluid hyaluronate $(H A)$ to hydroxyl radicals (bold line) compared with two hyaluronate standards. Experimental conditions were as in fig $2 C$, but the TSK 5000 PW column was used to obtain better molecular weight resolution. Flow was $0.5 \mathrm{ml} / \mathrm{min}$.

about 20.5 minutes, corresponding with the highly depolymerised hyaluronate. Figure 3 shows the molecular weight determination and calibration of the column. The hyaluronate degradation product induced by hydroxyl radicals was chromatographed with a TSK 5000 PW column (flow $0.5 \mathrm{ml} / \mathrm{min}$ ), which was more suitable for molecular weight determinations of small molecular size hyaluronate (fig 3 ). The molecular weight varied between 10.5 and

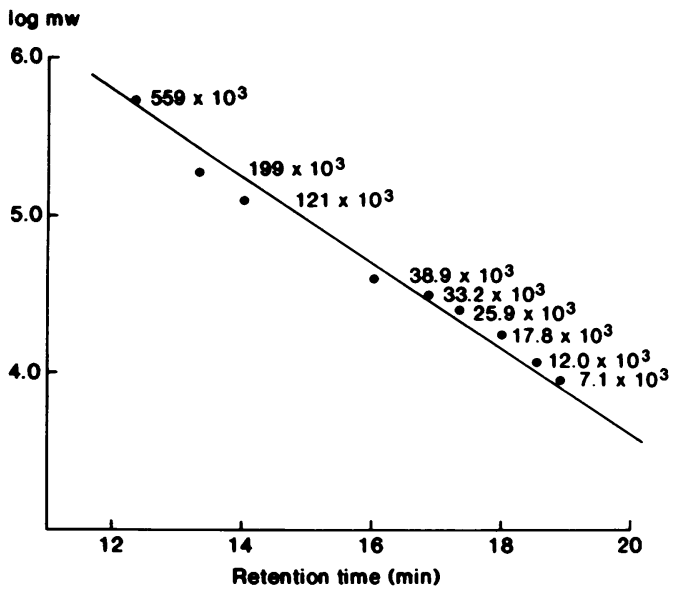

Figure 4 Molecular weight calibration of the TSK 5000 $P W$ column with hyaluronate standards. Each point represents a separate chromatogram. Flow was $0.5 \mathrm{ml} / \mathrm{min}$.

Viscometric measurements of synovial fluids containing different additives. ${ }^{*}$ Results are given as a percentage of the initial viscosity

\begin{tabular}{ll}
\hline Additives & $\begin{array}{l}\text { Viscosity } \\
\text { after } 60 \text { min }(\%)\end{array}$ \\
\hline $\begin{array}{l}\text { HX, XAO+ } \\
\text { Fe-EDTA }\end{array}$ & $22 \cdot 3$ \\
HX, XAO \\
$\begin{array}{l}\text { Desferrioxiamine } \\
\text { Mannitol }\end{array}$ \\
$\begin{array}{l}\text { HX } \\
\text { Desferrioxiamine } \\
\text { Mannitol }\end{array}$ \\
$\begin{array}{l}\text { HX, XAO } \\
\text { Fe-EDTA } \\
\text { Superoxide dismutase } \\
\text { HX, XAO } \\
\text { Fe-EDTA } \\
\text { Catalase }\end{array}$ \\
\hline
\end{tabular}

* A synovial fluid sample obtained from a patient with meniscus lesion was divided into five test tubes with additives, shown in lesion was divided into five test tubes with additives, shown in the table. After measurement of relative viscosities the reaction incubation the viscosities were measured again. †HX=hypoxanthine; $\mathrm{XAO}=$ xanthine oxidase.

$15.0 \times 10^{3}$ daltons in different experiments, the average being $13.5 \times 10^{3}$ daltons. Figure 4 shows the molecular weight calibration curve.

When $1 \mathrm{mM}$ desferrioxamine and $80 \mathrm{mM}$ mannitol were then added to the synovial fluid sample before exposure to xanthine oxidase/ hypoxanthine the hyaluronate peak diminished without a change in retention time, but the ${ }^{125} \mathrm{I}$ HABP peak increased, probably because configurational changes of the synovial fluid hyaluronate liberated new binding sites for the ${ }^{125}$ I labelled hyaluronate binding protein.

Viscometric measurements showed that superoxide dismutase and catalase eliminated the effect of oxygen derived free radicals almost completely (table).

\section{Discussion}

In non-inflammatory synovial fluid the high viscosity is mainly due to hyaluronate. In synovial fluid from patients with rheumatoid arthritis the viscosity is greatly decreased. It has been suggested that physicochemical changes characteristic of inflammatory synovial fluid- 
that is, low viscosity and depolymerisation of hyaluronate, might result from radical reactions. ${ }^{810}$ This paper describes a rapid, sensitive method for studying degradation products of synovial fluid hyaluronate induced by oxygen derived free radicals using HPLC with a size exclusion column.

Production of hydroxyl radicals may be necessary for depolymerisation of hyaluronate. ${ }^{11} 12$ Superoxide radicals and hydrogen peroxide themselves can also react with synovial fluid hyaluronate, however. Addition of desferrioxamine and mannitol ensures that only superoxide radicals, and hydrogen peroxide resulting from the spontaneous dismutation of superoxide radicals, are generated in the xanthine oxidase/hypoxanthine system.

It was noted that the synovial fluid hyaluronate HPLC peak decreased but the molecular size was almost unchanged. It may be that superoxide radical has a limited 'hyaluronatease' mimicking action, resulting in the formation of degradation products smaller than decasaccharides and thus not detectable by the ${ }^{125} \mathrm{I}$ HABP assay. ${ }^{13}$ At the same time the molecular configuration of synovial fluid hyaluronate is changed in such a way that new bindings sites for the ${ }^{125}$ I-HABP are freed, as shown by the increase in the ${ }^{125} \mathrm{I}-\mathrm{HABP}$ assay hyaluronate peak. Alternatively, the apparent effects of superoxide radicals and hydrogen peroxide might be caused by hydroxyl radicals formed locally, catalysed by iron bound to synovial fluid hyaluronate itself. This would cause reaction of hydroxyl radicals at the point at which they were formed, leading to degradation of hyaluronate. If this were the case this endogenous iron must be sterically located so that desferrioxamine and mannitol are excluded by hyaluronate and thus unable to prevent the localised Haber-Weiss reaction.

Several attempts have been made to find degradation products of small molecular size in inflamed synovial fluid. No such compounds, comparable with the hydroxyl radical end product shown in figs $2 \mathrm{C}$ and 3 have been found. ${ }^{14}$ Clearance and further degradation of hyaluronate degradation products of small molecular size might be so rapid that they are not detected in inflamed synovial fluid. Also, in vivo production of oxygen derived free radicals by polymorphonuclear neutrophilic leucocytes is intermittent, occurring during a respiratory burst in the ischaemia-reperfusion syndrome, and nor quite so effective as the continuous free radical flux produced in vitro by the xanthine oxidase/hypoxanthine system. ${ }^{15} 16$ It therefore seems that the progressive degradation of synovial fluid hyaluronate upon exposure to hydroxyl radicals, seen in HPLC recordings, represents the extreme molecular size ranges in which synovial fluid hyaluronate can exist. In practice, the molecular weight of hyaluronate in synovial fluid samples is located somewhere between the extreme limits, as shown by earlier HPLC studies. High performance liquid chromatograms of synovial fluids, obtained from patients with meniscus lesions or different forms of arthritis, show a synovial fluid hyaluronate molecular size distribution close to that seen in figs $1 \mathrm{~A}$ and $\mathrm{B} .{ }^{9}$

The simultaneous determination of the degree of polymerisation and the concentration of synovial fluid hyaluronate by HPLC makes it a useful tool in the study of oxygen derived free radical induced degradation of synovial fluid hyaluronate in inflammatory joint diseases.

1 Babior B M, Kipnes R S, Curnette J T. Biological defence mechanisms: the production by leukocytes of superoxide: a

2 Johnston J B, Lehmeyer J E, Guthrie L A. Generation of superoxide anion and chemiluminescence by human monocytes during phagocytosis and on contact with surface cytes during phagocytosis and on contact with surface
bound immunoglobulin. $\mathcal{F}$ Exp Med 1976; 143: 1551-6.

3 Halliwell B. Superoxide-dependent formation of hydroxyl radicals in the presence of iron salts. FEBS Lett 1978; 96: radicals

4 Fridovich I. Quantitative aspects of the production of superoxide anion radical by milk xanthine oxidase. $\mathcal{F}$ Biol Chem 1979; 245: 4053-7.

5 Haber F, Weiss J. The catalytic decomposition of hydrogen peroxide by iron salts. Proceedings of the Royal Society of London [A] 1934; 147: 332.

6 McCord J M. Free radicals and inflammation: protection of synovial fluid by superoxide dismustase. Science 1974; 185: 529-31.

7 Salin $M$ L, McCord J M. Free radicals and inflammation. J Clin Invest 1975; 56: 1319-23.

8 Greenwald R A, Moy W W. Effect of oxygen-derived radicals on hyaluronic acid. Arthritis Rheum 1980; 23: 455-63.

9 Saari H T, Konttinen Y T. Determination of synovial fluid hyaluronate concentration and polymerisation by high performance liquid chromatography. Ann Rheum Dis 1989; 48: $565-70$.

10 McNeil J D, Wiebkin O W, Betts W H, Cleland L G. Depolymerization products of hyaluronic acid after exposure to oxygen derived free radicals. Ann Rheum Dis 1985; 44: 780-9.

11 Carlin G, Djursäter R. Xanthine oxidase induced depolymerization of hyaluronic acid in the presence of ferritin FEBS Lett 1984; 177: 27-30.

12 Rowley D A, Halliwell B. Formation of hydroxyl radicals from hydrogen peroxide and iron salts by superoxide- and from hydrogen peroxide and iron salts by superoxide- and logy of rheumatoid arthritis. Clin Sci 1983; 64: 649-53.

13 Brandt $\mathbf{R}$, Hedlöf $\mathbf{E}$, Bucht A, Tengblad A. A convenient radiometric assay for hyaluronan. Acta Otolaryngol Suppl (Stockh) 1987; 442: 31-5.

14 Saari H T, Konttinen Y T, Santavirta S. Synovial fluid hyaluronate: a study using high performance liquid chromatography with size exclusion column. Medical Science Research 1989; 17: 99-101.

15 Woodruff T, Blake D R, Freeman J, Andrews F J, Salt P, Lunec $J$. Is chronic synovitis an example of reperfusion injury? Ann Rheum Dis 1986; 45: 608-11.

16 Greeneald R A, Moak S A. Degradation of hyaluronic acid by polymorphonuclear leukocytes. Inflammation 1986; 10: polymo. 